\title{
Generation of discrete structures in phase-space via charged particle trapping by an electrostatic wave
}

\author{
Dmitri Vainchtein ${ }^{1,2}$, Greg Fridman ${ }^{1}$, Anton Artemyev ${ }^{3,2}$ \\ ${ }^{1}$ Nyheim Plasma Institute, Drexel University, Camden, NJ, USA \\ ${ }^{2}$ Space Research Institute, Moscow, Russia \\ ${ }^{3}$ Institute of Geophysics and Planetary Physics, University of California, Los Angeles, CA, USA
}

\begin{abstract}
The wave-particle resonant interaction plays an important role in the charged particle energization by trapping (capture) into resonance. For the systems with waves propagating through inhomogeneous plasma, the key small parameter is the ratio of the wave wavelength to a characteristic spatial scale of inhomogeneity. When that parameter is very small, the asymptotic methods are applicable for the system description, and the resultant energy distribution of trapped particle ensemble has a typical Gaussian profile around some mean value. However, for moderate values of that parameter, the energy distribution has a fine structure including several maxima, each corresponding to the discrete number of oscillations a particle makes in the trapped state. We explain this novel effect which can play important role for generation of unstable distributions of accelerated particles in many space plasma systems.
\end{abstract}

Keywords: wave-particle interaction; resonance; adiabatic invariant

\section{Introduction}

Wave-particle resonant interaction in strong magnetic fields explains many observable effects in space plasma systems [1,2]. For sufficiently intense waves, this interaction is nonlinear, [3, 4] and includes such effects as phase trapping (capture) and phase bunching. Natural inhomogeneity of plasma and magnetic fields in the near-Earth space (radiation belts, the outer magnetosphere, solar wind) results in significant modification of resonant particle interaction $[5,6,7]$. Particle trapping in inhomogeneous plasma can lead to formation of unstable plasma distributions and emission of secondary waves $[8,9,10]$. This theory is widely used for description of wave generation in the Earth's radiation belts $[11,12,13,14,15]$.

Dynamics of trapped particles is defined by a (small) parameter of the plasma system with a wave: the ratio of wavelength $\sim 1 / k$ and background plasma inhomogeneity scale $L: \kappa=1 / k L$. For the nonlinear wave-particle interaction to be important, the wave amplitude $\Phi_{0}$ should be of order of $\kappa$ (or larger), otherwise wave electromagnetic fields are too weak to significantly affect charged particle motion $[3,4,16]$. Intense waves generate effective potential wells where particles can be trapped. Trapped particles oscillate in the effective potential with a frequency $\Omega_{\text {trap }} \sim$ $\sqrt{1 / K}$ (see, e.g., $[17,18,19]$ ), whereas the duration of the trapped motion is of order of $O(1)$, i.e., it does not depend on $\kappa$. Thus, trapped particles make $N \sim \sqrt{1 / \kappa}$ oscillations between the instance of trapping (capture) into resonance and escape from resonance. For $N \gg 1$ asymptotic theory adequately predicts change of particle energy between trapping and escape $[20,21,22,23,24]$. In this case, the final particle spectrum does not depend on $N$ (i.e., on $\kappa$ ). However, for many realistic systems (e.g., electron trapping by kinetic Alfven waves [25] and very oblique whistler waves [26, 27] in the equatorial Earth magnetosphere, electron trapping by Langmuir waves in solar wind [28], ion trapping by ion-cyclotron wave [29]) the number $N$ can be about $1-10$, i.e., particles escape from the resonance (e.g., due to significant variation of the wave amplitude along the wave path or due to perturbations of the trapped motion [30]) after just a few oscillations. In this case, the asymptotic theory of trapped particle motion is not well justified and new effects can appear in such systems.

Effects of finite $N$ on the wave-particle nonlinear interaction are beyond the scope of asymptotic theory $(\kappa \rightarrow 0)$ and requires consideration of peculiarities of the trapped motion in the phase plane. During the trapped particle 
motion, particle energy (or momentum) changes slowly at a rate $\sim O(1)$. Therefore, change of particle energy between the $N-1$-th and the $N$-th oscillations is about $\sim 1 / \Omega_{\text {trap }} \sim \sqrt{\kappa}$. For systems with $\kappa \rightarrow 0$, there is no essential difference of energy gained by particles making $N$ and $N-1$ oscillations. However, for small $N$ (moderate $\kappa$ ), particles escaping from the resonance after $N$ and $N-1$ oscillations can be significantly separated in terms of energy or physical space. Therefore, the separation between particles making different number of oscillations provides final particle spectrum with a discrete structure. Formation of the discrete spectrum with several maxima from wave-particle interaction is important both for further instability of this spectrum with corresponding emission of secondary waves and for estimate of maximum energies gained by trapped particle.

In the present paper, we consider a simplified plasma system where an electrostatic wave propagates along an inhomogeneous magnetic field. We approximate the magnetic field inhomogeneity by a parabolic shape, thus the system configuration can describe wave-particle interaction in the equatorial Earth's inner magnetosphere [24, 25, 31]. A similar configuration describes the classical system of the Langmuir wave damping in a weak transverse magnetic field in relativistic [32, 33, 34] and non-relativistic [35, 36] systems. We examine effects of finite $N$ on the redistribution of trapped electrons in the phase space.

\section{Main equations}

We consider charged particle (charge $e$, mass $m$ ) motion in a background magnetic field $B_{0}(z) \mathbf{e}_{z}$ and a field of an electrostatic wave $\Phi_{0} \sin \phi$ propagating along the $z$-axis: $\phi=k z-\Omega t$ and $\Phi_{0}$ is a wave amplitude. As the wave phase does not depend on the transverse coordinates, the system has $3 / 2$ degrees of freedom: conjugated variables $\left(z, p_{z}\right)$ and time $t$. Thus, the particle magnetic moment is conserved, $\mu=$ const, and the Hamiltonian can be written as [37]:

$$
H_{z}=\frac{1}{2 m} p_{z}^{2}+\mu B_{0}+e \Phi_{0} \sin \phi(t, z)
$$

We approximate the magnetic field by a parabolic profile: $B_{0}=B_{e q}\left(1+z^{2} / 2 L^{2}\right)$ where $B_{e q}$ is an amplitude value and $L$ is a spatial scale of magnetic field variation. Thus, Hamiltonian $H_{z} \rightarrow H_{z}-\mu B_{e q}$ can be written as

$$
H_{z}=\frac{1}{2 m} p_{z}^{2}+\frac{1}{2} m \Omega_{0}^{2} z^{2}+e \Phi_{0} \sin \phi(t, z)
$$

where $\Omega_{0}=\sqrt{\mu B_{e q} / m L^{2}}=$ const is a particle bounce frequency. We introduce following dimensionless variables and parameters: $\kappa=1 / k L, z_{1}=z k, p_{z 1}=p_{z} / L \Omega_{0} m, H_{1}=H_{z} / m L^{2} \Omega_{0}^{2}, t_{1}=t \Omega_{0} / \kappa, \kappa \varepsilon=e \Phi_{0} / \mu B_{e q}, \omega=\kappa \Omega / \Omega_{0}$ :

$$
\begin{aligned}
H_{1} & =\frac{1}{2} p_{z 1}^{2}+\frac{1}{2} \kappa^{2} z_{1}^{2}+\varepsilon \kappa \sin \phi_{1}\left(t_{1}, z_{1}\right) \\
\phi_{1} & =z_{1}-\omega t_{1}
\end{aligned}
$$

We assume that the wave length is much smaller than the inhomogeneous scale $(\kappa \ll 1)$, the wave phase velocity $k \Omega$ is about the particle thermal velocity $\Omega_{0} L$ (i.e., $\omega \sim 1$ ), and the wave energy is much smaller than the particle kinetic energy, $e \Phi_{0} / \mu B_{e q} \sim \kappa$, which means that $\varepsilon \sim 1$. We introduce new variables $\left(\phi, P_{\phi}\right),\left(x, P_{x}\right)$ using generating function $F_{2}=P_{\phi}\left(z_{1}-\omega t\right)-\kappa x z_{1}$ (note, $P_{x}$ is a new coordinate and $x$ is a conjugated momentum):

$$
\begin{aligned}
H & =H_{1}+\frac{\partial G_{1}}{\partial t}=H_{1}-\omega P_{\phi} \\
p_{z 1} & =\frac{\partial G_{1}}{\partial z_{1}}=P_{\phi}-\kappa x, \quad P_{x}=\frac{\partial G_{1}}{\partial x}=-\kappa z_{1} \\
\phi & =\frac{\partial G_{1}}{\partial P_{\phi}}=z_{1}-\omega t_{1}
\end{aligned}
$$

There is one additional equation for momentum conjugated to $t$, but the new Hamiltonian $H$ does depend on $t$, and thus the corresponding momentum is conserved and can be set to zero. Note that now $\phi$ is an independent variable. New Hamiltonian takes the form:

$$
H=-\omega P_{\phi}+\frac{1}{2} P_{x}^{2}+\frac{1}{2}\left(P_{\phi}-\kappa x\right)^{2}+\varepsilon \kappa \sin \phi
$$



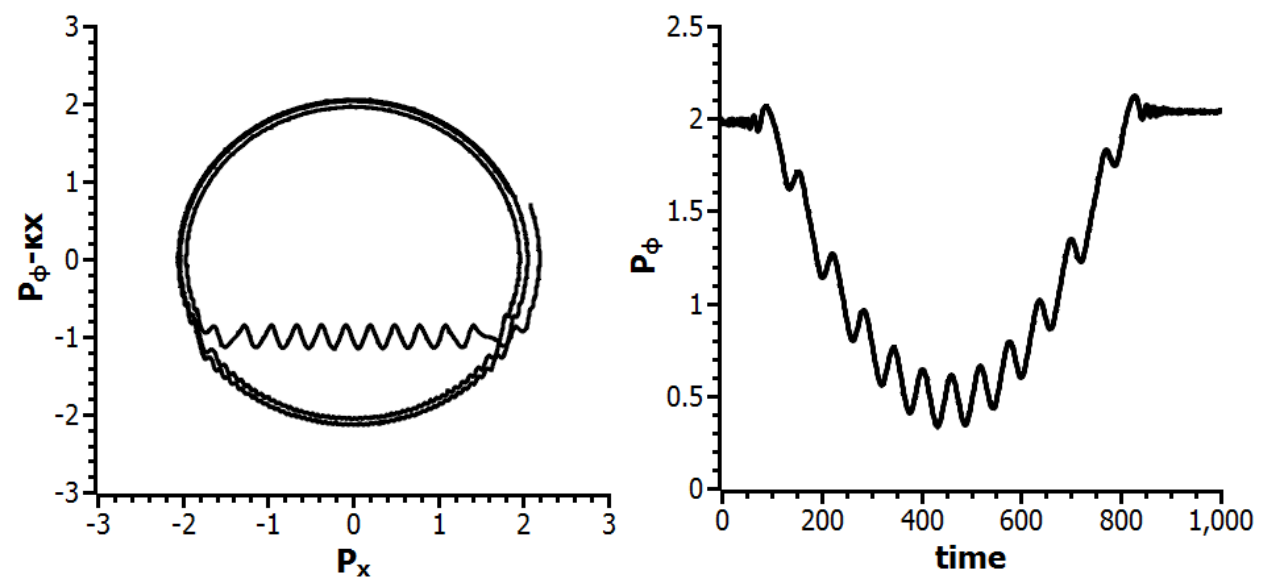

Figure 1: Characteristic particle trajectory in the presence of trapping: in the $\left(P_{\phi}-\kappa x, P_{x}\right)$ plane (left panel), and the corresponding evolution of $P_{\phi}$ as a function of time (right panel) for the part of the trajectory around trapping. Parameters are $\kappa=0.005, \varepsilon=3, \omega=1$. The trajectory is obtained by numerical integration of Eqs. (6) with the Runge-Kutta method.

The corresponding Hamiltonian equations are

$$
\begin{aligned}
\dot{x} & =P_{x} \\
\dot{P}_{x} & =\kappa\left(P_{\phi}-\kappa x\right) \\
\dot{\phi} & =-\omega+\left(P_{\phi}-\kappa x\right) \\
\dot{P}_{\phi} & =-\varepsilon \kappa \cos \phi
\end{aligned}
$$

In System (6), $\left(\kappa x, P_{x}, P_{\phi}\right)$ are slow variables (change rate is of order of $\left.\kappa\right)$ and $\phi$ is a fast phase. Note that Hamiltonian $H_{z}$ in (1) and (2) is not conserved, as it depends explicitly on time. On the other hand, Hamiltonian $H$ in (5) is conserved. As the two Hamiltonians differ (up to a coefficient) by the quantity $\omega P_{\phi}$, changes in $P_{\phi}$ are equivalent to changes in the energy of particles:

$$
H_{z}=\text { const }+m L \Omega_{0}(\Omega / k) P_{\phi}
$$

where $P_{\phi}$ is dimensionless.

\section{Resonant interaction}

Particles oscillate in the $\left(\kappa x, P_{x}\right)$ plane of the slow variables and may become trapped by the wave. Equations (6) show that the resonant condition $\dot{\phi}=0$ corresponds to the line $P_{\phi}=P_{\phi, r}=\omega+\kappa x$, see Fig. 1. When plotting Fig. 1 and everywhere else in the paper we used the following values of the parameters: $\kappa=0.005, \varepsilon=3, \omega=1$. Trapping occurs at a negative $P_{x, \text { trap }}$ and, due to the system's symmetry in the $\left(\kappa x, P_{x}\right)$ plane, particles escape from the resonance at a positive $P_{x, e s c} \approx-P_{x, \text { trap }}$. Thus $P_{\phi}$ at the moment of escape equals to $P_{\phi}$ at the moment of trapping (for trajectory shown in Fig. 1, the initial $P_{\phi}=2$ ). More precisely, the exit values of $P_{\phi}$ are expected to have a Gaussian distribution about a mean value $P_{\phi} \approx 2$. However, numerical simulations indicate the presence of several maxima, see Fig. 2 where we numerically integrated $10^{6}$ trajectories of (6) with the same initial values of $\left(x, P_{x}, P_{\phi}\right)$ but different initial phases $\phi_{0}$. We considered only trajectories with the trapping/escape event and plotted the distribution of $P_{\phi}$ after each particle escaped from the resonance. Note the semi-log scale: there is an order of magnitude difference in the number of particles at the two rightmost maxima compared with the valley between them.

To study the resonant interaction in detail, we expand Hamiltonian (5) around the resonant value of $P_{\phi}=P_{\phi, r}$ :

$$
H=\frac{1}{2}\left(P_{\phi}-P_{\phi, r}\right)^{2}-\omega P_{\phi, r}+\frac{1}{2} P_{x}^{2}+\frac{1}{2} \omega^{2}+\varepsilon \kappa \sin \phi
$$




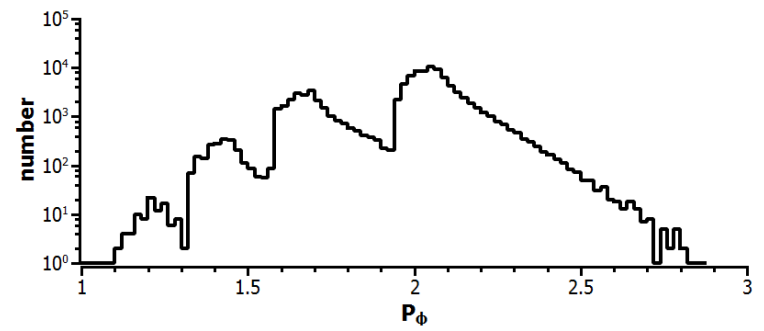

Figure 2: Distribution of $P_{\phi}$ after escape from the resonance. All particles have the same initial $P_{\phi}=2$.
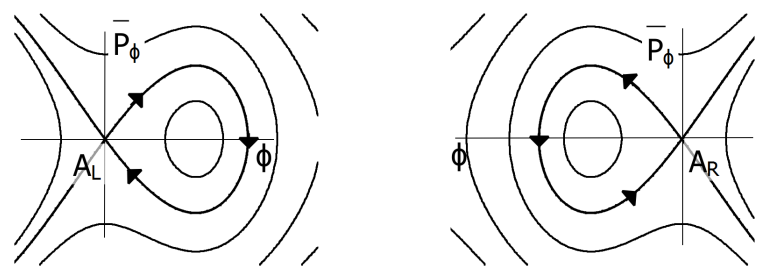

Figure 3: Schematic phase portraits of Hamiltonian $H_{\phi}$ in the resonance phase plane. Left: $P_{x}>0$, Right: $P_{x}<0$.

Using a generating function $G_{2}=\left(P_{\phi}-P_{\phi, r}\right) \bar{\phi}+P_{x} \bar{x}$, we can introduce new conjugated variables $\left(\bar{P}_{\phi}, \bar{\phi}\right),\left(\bar{P}_{x}, \bar{x}\right)$ :

$$
\begin{aligned}
& \bar{P}_{\phi}=P_{\phi}-P_{\phi, r}, \quad \bar{\phi}=\phi \\
& \bar{P}_{x}=P_{x}-\kappa \bar{\phi}, \quad \bar{x}=x
\end{aligned}
$$

and the Hamiltonian takes the form $H=H_{x}+H_{\phi}$

$$
\begin{aligned}
& H_{x}=\frac{1}{2} P_{x}^{2}-\omega \kappa x-\frac{1}{2} \omega^{2} \\
& H_{\phi}=\frac{1}{2} \bar{P}_{\phi}^{2}+\kappa P_{x} \phi+\varepsilon \kappa \sin \phi
\end{aligned}
$$

where we omitted the bars over the $\left(\kappa x, P_{x}, \phi\right)$ variables. Hamiltonian $H_{x}$ describes the evolution of slow variables $\left(x, P_{x}\right)$ and Hamiltonian $H_{\phi}$ describes the fast oscillations in the $\left(\phi, \bar{P}_{\phi}\right)$ plane with a slowly changing parameter $P_{x}$. Phase portraits of Hamiltonian $H_{\phi}$ are shown in Fig. 3. Hamiltonian $H_{\phi}$ has two fixed points. For $P_{x}<0$ the hyperbolic fixed point is to the right of the elliptic point, and for $P_{x}>0$ the hyperbolic fixed point is to the left of the elliptic point (recall that $P_{x}$ changes sign in the process of the trapped motion).

\section{Trapped dynamics in detail}

As the first step in interpreting the distribution in Fig. 2, recall that the only discrete property of the trapped dynamics is the number of oscillations a particle makes in the resonance. Figure 4 illustrates that the final value of $P_{\phi}$ strongly correlates with the number of oscillations a particle makes in the resonance. The distribution contains several maxima, each with an exponential tail to the right and a relatively sharp drop to the left. It is the objective of the present paper to explain this phenomenon.

First, we can discuss the distances between the maxima in Fig. 4. Figure 5 shows characteristic trajectories corresponding to different number of oscillations in the resonance. Upon the arrival into resonance, a particles starts oscillating in the resonance phase plane. The particles enter and leave the resonance at the top of the separatrix loop in Fig. 3. By integrating the term for $\dot{P}_{\phi}$ in Eq. (6), we obtain that the change of $P_{\phi}$ over a full oscillation in the resonance is proportional to the value of $P_{x}$ during that oscillation. In essence, as one can see from Fig. 5, the larger is the value of $P_{x}$ at the release, the larger is the value of the gain of $P_{\phi}$ during the last oscillation in trapping. On 


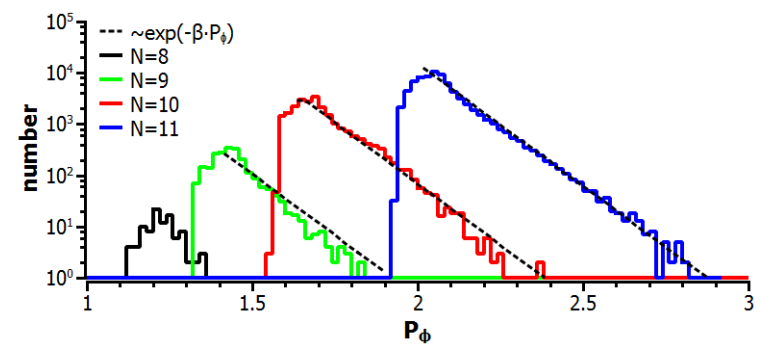

Figure 4: Histogram of the exit distribution of $P_{\phi}$ for different number of oscillations. Note the semi-log sale.
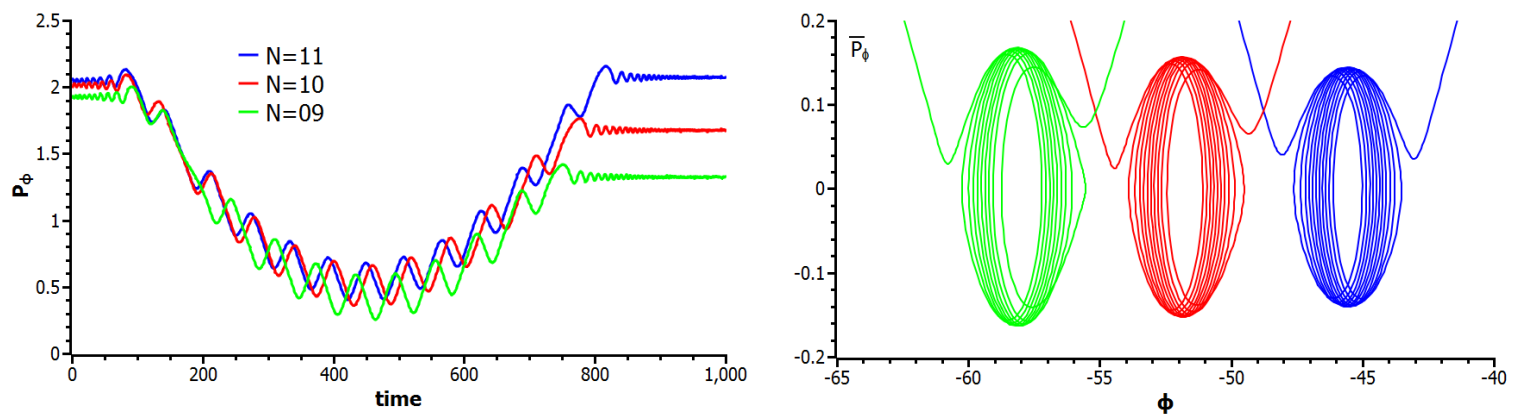

Figure 5: Typical phase trajectories in capture (a) Evolution of $P_{\phi}$; (b) Projection on the $\left(\phi, \bar{P}_{\phi}\right)$ plane.

the second half of trapping (past the line $P_{x}=0$ in Fig. 1) with every oscillation the value of $P_{x}$ increases. Thus, the more oscillations a particle makes in the resonance, the larger is the value of $P_{x}$ at the release. Therefore, the distance between the maxima $N=11$ and $N=10$ is larger than the distance between the maxima $N=10$ and $N=9$.

Most of the particles do not approach either of two hyperbolic fixed points, and, after making a certain number of oscillations, exit the resonance. We are interested in the trajectories that differ from that standard path. It is the trajectories that approach one (or both) of the fixed points that modify the final distribution. In short, the fixed point at the right, $A_{R}$, is responsible for reducing the number of oscillations and the mean value of $P_{\phi}$ at the exit, while the fixed point at the left, $A_{L}$, is responsible for increasing the mean value of $P_{\phi}$ at the exit while not affecting the number of oscillations.

The number of particles in each of the two rightmost maxima, which contain most of the particles, depend more on the particular choice of the initial conditions and the fine details of the values of the parameters of the problem. It is the number of particles is the subsequent maxima that are defined by the fixed points.

A particle may come close to the right fixed point, either on the last oscillation outside the separatrix $S$, or on the first oscillation inside $S$, but only once. As the outcome is the same in the both cases, let's assume that the approach happens inside the region surrounded by $S$. Near $A_{R}$ the particle slows down and the equations of motion are reduced to

$$
\begin{aligned}
& \dot{P}_{\phi}=-\varepsilon \kappa \cos \phi_{R} \approx \kappa P_{x} \\
& \dot{P}_{x} \approx \kappa \omega
\end{aligned}
$$

where $\phi_{R}$ is a coordinate of $A_{R}$ point. Therefore, instead of oscillations, the value of $P_{\phi}$ decreases "ballistically" while a particle is stuck near $A_{R}$ as $P_{x}<0$ (see the part of the black curve towards time $=200$ in Fig. 5). The sharper is the corner at the right of the oscillations in Fig. 5 (compare the black curve with the other two), the closer a particles comes to $A_{R}$. Similarly, while the particle is there, the value of the area under $S, \Sigma=\Sigma\left(P_{x}\right)$, is increasing. The longer a particle stays near $A_{R}$, the smaller the value of $P_{\phi}$ and the larger the value of $\Sigma$ becomes. (Note, that when $P_{x}$ reaches the value $P_{x}=0, A_{R}$ seize to exist, the value of $\Sigma$ reaches the maximum, and particles naturally proceed with their motion and are released from resonance immediately). Effectively, the particles are released with the values of $P_{\phi}$ and 
$\Sigma$ not with which they crossed $S$, but with which they completed the first oscillation. The black trajectory in Fig. 5 is an example of a trajectory that comes close to $A_{R}$.

After the first oscillation is done, the number of oscillations is set, and the minimal value of $P_{\phi}$ is also defined, unlike the maximum possible value, which can be changed by a close approach to $A_{L}$.

After completing a certain number of oscillations, on the last oscillation before coming out of $S$ (or on the first oscillation after crossing $S$ ), a particle may come close to $A_{L}$. Near $A_{L}$ the dynamics is described by the same Eq. (11), as near $A_{R}$, but with $P_{x}>0$. Therefore, it leads to the increase of the value of $P_{\phi}$. However, the number of oscillations does not change: the oscillation was the last one anyway (or the particle was even outside $S$ already). Therefore, a close approach to $A_{L}$ results in the appearance of the tails into large values of $P_{\phi}$ of the distributions in Fig. 4 . As the additional increase of $P_{\phi}$ is essentially proportional to the time spent near $A_{L}$, which, in turn, is logarithmic in distance from $A_{L}$, we expect an exponential decay of the tails.

The time a particle spends near $A_{L}$ is

$$
T \approx \frac{1}{\sqrt{\varepsilon \kappa \sin \phi_{L}}} \ln \left(\phi-\phi_{L}\right)+\text { const }
$$

where $\phi_{L}$ is the value of $\phi$ at $A_{L}$. Assuming that $P_{x}$ does not change too much while a particle is near $A_{L}$ and using Eq. (11), we get for the excess value (compared with the "expected" value at the maximum of the corresponding distribution in Fig. 4)

$$
\Delta P_{\phi} \approx \sqrt{\kappa} \beta \ln \left(\phi-\phi_{L}\right), \quad \beta=P_{x, 0} / \sqrt{\varepsilon \sin \phi_{L}}
$$

where $P_{x, 0}$ is the value of $P_{x}$ during the approach to $A_{L}$. Assuming that the values of $\phi-\phi_{L}$ are distributed uniformly, we obtain the slope of the probability distribution function $M=M\left(\Delta P_{\phi}\right)$ to the right of any given maximum $M_{0}$ as

$$
M\left(\Delta P_{\phi}\right) \approx M_{0} \exp \left(-\Delta P_{\phi} /(\sqrt{\kappa} \beta)\right)
$$

Figure 4 shows that results of numerical calculations confirm approximation (14).

Unlike increasing the value of $P_{\phi}$ at the exit, there is no similar way to drastically decrease it. Indeed, after the completion of the first oscillation when the total number of loops is set, there is a maximum value by which $P_{\phi}$ can decrease while $P_{x}<0$ and a minimum value by which $P_{\phi}$ can increase while $P_{x}>0$. Therefore, for each value of the number of oscillations, there is a well-defined minimum possible value of $P_{\phi}$.

\section{Discussion and conclusions}

Once recalled that the adiabatic invariant $P_{\phi}$ is essentially the energy (see (7)), Fig. 4 clearly demonstrates that for systems with a relatively few oscillations in resonance $(N \sim 10)$, the distribution of the energy of particles upon the escape from resonance has several well separated maxima. This novel effect can play important role for emission of secondary waves. Indeed, the final spectrum contains many localised maxima with corresponding positive slopes $d M / d P_{\phi}>0$ of the distribution function. The growth of waves resonantly interacting with particle distribution and having $d M / d P_{\phi}>0$ results in the generation (or amplification) of a discrete spectrum of waves. One possible example of such a system was observed by spacecraft in the near-Earth plasma space: electron trapping into resonance with a strong whistler wave results in the formation of the emission of secondary waves with a discrete spectrum [38]. Another example corresponds to spacecraft observations of electron trapping into resonance with an ultra-low frequency wave, when the wave-particle resonant interaction results in the formation of a discrete energy spectrum [39]. For this system, the proposed mechanism of the discrete spectrum formation could operate together with the mechanisms proposed in [40].

Equation (7) shows that difference in energy of particles escaping from resonance after $N$ and $N-1$ oscillations is about $\Delta H_{z} \sim\left(m L \Omega_{0} \Omega / k\right) \Delta P_{\phi}$ where $\Delta P_{\phi} \sim \sqrt{\kappa \varepsilon}$ (about resonance width [19]). Introducing wave phase velocity $v_{\phi}=\Omega / k$ and using an estimate $\mu \sim \Omega_{0}^{2} L^{2} m / 2 B_{e q}$, we obtain $\Delta H_{z} \sim \sqrt{m v_{\phi}^{2} e \Phi_{0}}$, i.e., the energy difference is of the same order as the resonant width in energy space [41]. For resonant particles, their velocity $\Omega_{0} L$ is of the order of the wave phase velocity: $\Delta H_{z} \sim H_{z} \sqrt{e \Phi_{0} / H_{z}} \sim H_{z} \sqrt{\kappa \varepsilon} \sim H_{z} N^{-1 / 2}$ (recall that dimensionless parameter $\varepsilon \sim$ 1). Therefore, the separation of particles in energy space is proportional to $N^{-1 / 2}$ and it should be detectable in 
systems with moderate $N$ and large energies of particles accelerated in trapping. For example, kinetic Alfven waves propagating in inhomogeneous magnetic field of the Earth's inner magnetosphere can trap $0.1 \mathrm{keV}$ electrons and accelerate them up to few keVs, whereas corresponding parameter $N \sim 1 / \kappa$ can as small as $10-100$ [25]. The corresponding final distributions of accelerated electrons can contain several maxima separated in energy space by few hundreds of eVs.

Analytical expression (14) and numerical results shown in Fig. 4 demonstrate that for moderate $\kappa$ the final distribution function of escaping particles has long exponential tails $\sim \exp \left(-\Delta P_{\phi} /(\sqrt{\kappa} \beta)\right)$. Therefore, a final value of energy predicted from asymptotic theory for $\kappa \rightarrow 0$ can be significantly exceeded. This effect is especially important for models predicting maximum energies of particles accelerated by nonlinear wave-particle interaction in the Earth's radiation belts $[11,12,13,14]$. Such models serve for forecasting of a possible damage inflicting by accelerated particles to artificial satellites [42]. Besides Space Plasma, the considered magnetic field configuration could be realized in a magnetic mirror machine developed for investigation of plasma production. Generation or injection of strong waves into mirror machines results in a strong wave-particle interaction. In this case, the excitation of sideband waves induced by particle trapping is observed in laboratory experiments [47, 48]. Potentially, the effects considered in our study can explain formation of such secondary (sideband) wave emissions.

In this study, we considered a fixed wave field and did not construct a complete self-consistent model of the waveparticle interaction. In a more general system, a self-consistent wave field modulation can lead phase mixing. The trapped-particle phase space would then become somewhat chaotic and any discrete phase-space structure might be smoothed out to some extend, [43].

The second effect related to self-consistency and omitted in our study is the energy exchange between particles and wave(s). There are two intermingled particle populations: trapped and transient (scattered by waves). In this study we focus on the evolution of trapped particles, but the total energy changes of trapped and transient (scattered) particles are generally comparable $[5,7]$. In many realistic systems, waves play a role of mediator between these two particle populations: transient particle transfer their energy to waves, and waves at the same time transfer their energy to trapped particles. Thus, typically transient particles decelerate, while trapped particles accelerate [44, 45, 46]. Therefore, the wave growth/damping is a second order effect corresponding to a possible small imbalance between energy transfers to trapped and from transient particles. This (nonlinear) regime of the wave growth/damping is much more complicated than the linear Landau damping and strongly depends on particle distribution functions and system asymmetries. That being said, in a symmetric geometry (with respect to the axis $P_{x}=0$ ) considered in our study, the total energy changes both for trapped and transient particles are equal to zero individually, and thus the wave energy is conserved.

To conclude, we considered a nonlinear wave-particle interaction in a system with a moderate value of the small parameter $\kappa$ (i.e., when the number of particle oscillations in trapping is $N \sim 10$ ). For such systems, peculiarities of the trapped motion of particles in the phase space can significantly influence the moment of particle escape from the resonance. In particular, the final distribution of particle energy $P_{\phi}$ has a several-maxima structure where each peak of this distribution corresponds to some value of $N$. Each peak within this distribution has an exponentially long tail $\sim \exp \left(-\Delta P_{\phi} /(\sqrt{\kappa} \beta)\right)$.

\section{Acknowledgments}

This material is based upon work supported by the National Science Foundation under Award No. CMMI-1362782 (D.V.) and on Russian Scientific Foundation, Project No. 14-12-00824 (A.A.).

\section{References}

\section{References}

[1] R. M. Thorne, W. Li, B. Ni, Q. Ma, J. Bortnik, L. Chen, D. N. Baker, H. E. Spence, G. D. Reeves, M. G. Henderson, C. A. Kletzing, W. S. Kurth, G. B. Hospodarsky, J. B. Blake, J. F. Fennell, S. G. Claudepierre, S. G. Kanekal, Rapid local acceleration of relativistic radiation-belt electrons by magnetospheric chorus, Nature 504 (2013) 411-414. doi:10.1038/nature12889.

[2] A. Y. Ukhorskiy, M. I. Sitnov, Dynamics of Radiation Belt Particles, Space Sci. Rev. 179 (2013) 545-578. doi:10.1007/s11214-012-9938-5.

[3] V. I. Karpman, Nonlinear Effects in the ELF Waves Propagating along the Magnetic Field in the Magnetosphere, Space Sci. Rev. 16 (1974) 361-388. doi:10.1007/BF00171564. 
[4] V. D. Shapiro, R. Z. Sagdeev, Nonlinear wave-particle interaction and conditions for the applicability of quasilinear theory, Physics Reports 283 (1997) 49-71. doi:10.1016/S0370-1573(96)00053-1.

[5] D. Shklyar, H. Matsumoto, Oblique Whistler-Mode Waves in the Inhomogeneous Magnetospheric Plasma: Resonant Interactions with Energetic Charged Particles, Surveys in Geophysics 30 (2009) 55-104. doi:10.1007/s10712-009-9061-7.

[6] J. M. Albert, X. Tao, J. Bortnik, Aspects of Nonlinear Wave-Particle Interactions, in: D. Summers, I. U. Mann, D. N. Baker, M. Schulz (Eds.), Dynamics of the Earth's Radiation Belts and Inner Magnetosphere, American Geophysical Union, 2013. doi:10.1029/2012GM001324.

[7] Y. Omura, D. Nunn, D. Summers, Generation Processes of Whistler Mode Chorus Emissions: Current Status of Nonlinear Wave Growth Theory, in: D. Summers, I. U. Mann, D. N. Baker, M. Schulz (Eds.), Dynamics of the Earth's Radiation Belts and Inner Magnetosphere, American Geophysical Union, 2013, pp. 243-254. doi:10.1029/2012GM001347.

[8] K. B. Dysthe, Some studies of triggered whistler emissions, J. Geophys. Res. 76 (1971) 6915-6931. doi:10.1029/JA076i028p06915.

[9] D. Nunn, A self-consistent theory of triggered VLF emissions, Planatary Space Science 22 (1974) 349-378. doi:10.1016/00320633(74)90070-1.

[10] V. I. Karpman, J. N. Istomin, D. R. Shklyar, Nonlinear theory of a quasi-monochromatic whistler mode packet in inhomogeneous plasma, Plasma Physics 16 (1974) 685-703. doi:10.1088/0032-1028/16/8/001.

[11] Y. Omura, Y. Katoh, D. Summers, Theory and simulation of the generation of whistler-mode chorus, J. Geophys. Res. 113 (2008) 4223. doi:10.1029/2007JA012622.

[12] Y. Omura, M. Hikishima, Y. Katoh, D. Summers, S. Yagitani, Nonlinear mechanisms of lower-band and upper-band VLF chorus emissions in the magnetosphere, J. Geophys. Res. 114 (2009) 7217. doi:10.1029/2009JA014206.

[13] A. G. Demekhov, V. Y. Trakhtengerts, Dynamics of the magnetospheric cyclotron ELF/VLF maser in the backward-wave-oscillator regime. II. The influence of the magnetic-field inhomogeneity, Radiophysics and Quantum Electronics 51 (2008) 880-889. doi:10.1007/s11141-0099093-3.

[14] A. G. Demekhov, Generation of VLF emissions with the increasing and decreasing frequency in the magnetosperic cyclotron maser in the backward wave oscillator regime, Radiophysics and Quantum Electronics 53 (2011) 609-622. doi:10.1007/s11141-011-9256-x.

[15] A. Osmane, L. B. Wilson, III, L. Blum, T. I. Pulkkinen, On the Connection between Microbursts and Nonlinear Electronic Structures in Planetary Radiation Belts, Astrophys. J. 816 (2016) 51. doi:10.3847/0004-637X/816/2/51.

[16] V. Y. Trakhtengerts, M. J. Rycroft, Whistler and Alfvén Mode Cyclotron Masers in Space, Cambridge University Press, 2008.

[17] D. Nunn, Wave-particle interactions in electrostatic waves in an inhomogeneous medium, Journal of Plasma Physics 6 (1971) 291. doi:10.1017/S0022377800006061.

[18] V. I. Karpman, D. R. Shklyar, Nonlinear Damping of Potential Monochromatic Waves in an Inhomogeneous Plasma, Sov. JETP 35 (1972) 500.

[19] P. J. Palmadesso, Resonance, Particle Trapping, and Landau Damping in Finite Amplitude Obliquely Propagating Waves, Physics of Fluids 15 (1972) 2006-2013. doi:10.1063/1.1693815.

[20] A. G. Demekhov, V. Y. Trakhtengerts, M. J. Rycroft, D. Nunn, Electron acceleration in the magnetosphere by whistler-mode waves of varying frequency, Geomagnetism and Aeronomy 46 (2006) 711-716. doi:10.1134/S0016793206060053.

[21] Y. Omura, N. Furuya, D. Summers, Relativistic turning acceleration of resonant electrons by coherent whistler mode waves in a dipole magnetic field, J. Geophys. Res. 112 (2007) 6236. doi:10.1029/2006JA012243.

[22] X. Tao, J. Bortnik, R. M. Thorne, J. M. Albert, W. Li, Effects of amplitude modulation on nonlinear interactions between electrons and chorus waves, Geophys. Res. Lett. 39 (2012) 6102. doi:10.1029/2012GL051202.

[23] A. Neishtadt, D. Vainchtein, A. Vasiliev, Dynamics of electrons in a parabolic magnetic field perturbed by an electromagnetic wave, Plasma Physics and Controlled Fusion 53 (8) (2011) 085014. doi:10.1088/0741-3335/53/8/085014.

[24] A. Artemyev, V. Krasnoselskikh, O. Agapitov, D. Mourenas, G. Rolland, Non-diffusive resonant acceleration of electrons in the radiation belts., Physics of Plasmas 19 (2012) 122901. doi:10.1063/1.4769726.

[25] A. V. Artemyev, R. Rankin, M. Blanco, Electron trapping and acceleration by kinetic Alfven waves in the inner magnetosphere, J. Geophys. Res. 120 (2015) 10. doi:10.1002/2015JA021781.

[26] A. Osmane, A. M. Hamza, Relativistic surfatron process for Landau resonant electrons in radiation belts, Nonlinear Processes in Geophysics 21 (2014) 115-125. arXiv:1402.1691, doi:10.5194/npg-21-115-2014.

[27] A. Artemyev, O. Agapitov, D. Mourenas, V. Krasnoselskikh, V. Shastun, F. Mozer, Oblique whistler-mode waves in the earth's inner magnetosphere: Energy distribution, origins, and role in radiation belt dynamics, Space Science Reviews 200 (2016) 261-355. doi:10.1007/s11214016-0252-5.

[28] C. Krafft, A. S. Volokitin, Electron Acceleration by Langmuir Waves Produced by a Decay Cascade, Astrophys. J. 821 (2016) 99. doi:10.3847/0004-637X/821/2/99.

[29] D. R. Shklyar, I. V. Kuzichev, Ion energization by ELF wave packets formed of lightning-induced emission in the low-altitude magnetosphere, Geophys. Res. Lett. 41 (2014) 201-208. doi:10.1002/2013GL058692.

[30] D. R. Shklyar, G. Zimbardo, Particle dynamics in the field of two waves in a magnetoplasma, Plasma Physics and Controlled Fusion 56 (9) (2014) 095002. doi:10.1088/0741-3335/56/9/095002.

[31] F. S. Mozer, O. Agapitov, A. Artemyev, J. F. Drake, V. Krasnoselskikh, S. Lejosne, I. Vasko, Time domain structures: What and where they are, what they do, and how they are made, Geophys. Res. Lett. 42 (2015) 36273638. doi:10.1002/2015GL063946.

[32] T. Katsouleas, J. M. Dawson, Unlimited electron acceleration in laser-driven plasma waves, Physical Review Letters 51 (1983) $392-395$. doi:10.1103/PhysRevLett.51.846.2.

[33] A. P. Itin, A. I. Neishtadt, A. A. Vasiliev, Captures into resonance and scattering on resonance in dynamics of a charged relativistic particle in magnetic field and electrostatic wave, Physica D: Nonlinear Phenomena 141 (2000) 281-296. doi:10.1016/S0167-2789(00)00039-7.

[34] A. P. Itin, Trapping and scattering of a relativistic charged particle by resonance in a magnetic field and an electromagnetic wave, Plasma Physics Reports 28 (2002) 592-603. doi:10.1134/1.1494058.

[35] R. Z. Sagdeev, V. D. Shapiro, Influence of transverse magnetic field on landau damping, Soviet Journal of Experimental and Theoretical Physics Letters 17 (1973) 279-282. 
[36] F. Valentini, P. Veltri, A. Mangeney, Magnetic-field effects on nonlinear electrostatic-wave Landau damping, Phys. Rev. E 71 (1) (2005) 016402. doi:10.1103/PhysRevE.71.016402.

[37] T. G. Northrop, The adiabatic motion of charged particles, Interscience Publishers John Wiley and Sons, New York-London-Sydney, 1963.

[38] X. Fu, Z. Guo, C. Dong, S. P. Gary, Nonlinear subcyclotron resonance as a formationmechanism for gaps in banded chorus, Geophys. Res. Lett. 42 (2015) 3150-3159. , doi:10.1002/2015GL064182.

[39] A. Y. Ukhorskiy, M. I. Sitnov, D. G. Mitchell, K. Takahashi, L. J. Lanzerotti, B. H. Mauk, Rotationally driven 'zebra stripes' in Earth's inner radiation belt, Nature 507 (2014) 338-340. doi:10.1038/nature13046.

[40] Y. Liu, Q.-G. Zong, X.-Z. Zhou, J. C. Foster, R. Rankin, Structure and evolution of electron "zebra stripes" in the inner radiation belt, J. Geophys. Res. 121 (2016) 4145-4157. doi:10.1002/2015JA022077.

[41] C. F. F. Karney, Stochastic ion heating by a lower hybrid wave, Physics of Fluids 21 (1978) 1584-1599. doi:10.1063/1.862406.

[42] R. B. Horne, S. A. Glauert, N. P. Meredith, D. Boscher, V. Maget, D. Heynderickx, D. Pitchford, Space weather impacts on satellites and forecasting the Earth's electron radiation belts with SPACECAST, Space Weather 11 (2013) 169-186. doi:10.1002/swe.20023.

[43] C. Villani, Particle systems and nonlinear Landau dampinga), Physics of Plasmas 21 (3) (2014) 030901. doi:10.1063/1.4867237.

[44] D. R. Shklyar, On the nature of particle energization via resonant wave-particle interaction in the inhomogeneous magnetospheric plasma, Annales Geophysicae 29 (2011) 1179-1188. doi:10.5194/angeo-29-1179-2011.

[45] I. Y. Vasko, O. V. Agapitov, F. S. Mozer, A. V. Artemyev, J. F. Drake, Electron holes in inhomogeneous magnetic field: Electron heating and electron hole evolution, Physics of Plasmas 23 (5) (2016) 052306. doi:10.1063/1.4950834.

[46] A. V. Artemyev, R. Rankin, I. Y. Vasko, Upper limit of electron fluxes generated by kinetic Alfven waves in maxwellian plasma, J. Geophys. Res. 121 (9) (2016) 8361-8373. doi:10.1002/2016JA023076.

[47] R. W. Clark, D. G. Swanson, P. Korn, F. Sandel, S. Robertson, C. B. Wharton, High power fundamental and harmonic resonant ion cyclotron heating in a mirror machine, Physics of Fluids 17 (6), (1974) 1322-1328. doi: 10.1063/1.1694884.

[48] H. Jhang, S. G. Lee, S. S. Kim, B. H. Park, J. G. Bak, Stabilization of Interchange Modes in Mirror Plasmas by a Nonlinear rf-Plasma Wave Coupling Process, Phys. Rev. Lett. 95 (2016), 035005. doi:10.1103/PhysRevLett.95.035005. 